\title{
TREATMENT OF CHRONIC TRICHOPHYTOSIS WITH LINDANE OINTMENT
}

\author{
MITSURU SHIRAKAWA \\ Department of Hygienics and Public Health, Kurume University School of \\ Medicine, Kurume-shi, Japan
}

The author confirmed experimentally a few years ago that the $\gamma$ isomer of BHC (Benzene hexachloride) had a curative effect on parasitic dermatosis such as scabies, trichophytosis, and pityriasis versicolor and that BHC had a fungicidal and fungal-growthinhibitory action on the trichophyton fungus. The BHC preparation was reexamined and improved later by the author and used in the treatment of various skin diseases for elucidation of its effect. The interesting result obtained will be described below.

I. Drug used. The drug used in the previous investigation was prepared by mixing crude BHC containing 7.7-50.0\% of its weight of $\gamma-\mathrm{BHC}$ with vanishing cream and contained the $\gamma$-isomer in concentrations of $1-2 \%$ together with a considerable amount of impurities and non-effective substances. The drug, particularly its $\delta$-isomer content, gave an irritative sensation when applied in certain diseases and was in need of further purification of its materials. Fortunately the recent mass production of Lindane, a highly purified preparation of $\gamma-\mathrm{BHC}$, has met this demand for an insecticide composed $99 \%$ or more of $\gamma$-BHC.

Beside, the drug used in the previously reported investigation, i. e. a mixture of minute particle of well-crushed BHC and vanishing cream, was attended with the possibility that the isomer might be decomposed by the cream which is, though slightly, alcaline. It was further feared that the drug might not be sufficiently permeable to be used in the treatment of eruptive trichophytosis which has its foci deep down in the skin.

In full consideration of the insufficiency of permeability and defect the drug to be used in the present experiment was prepared as follows (see Table 1):-

Materials : Polyethylene glycol (compound 2200) (Dainippon Seiyaku), hydrophilic ointment (Sankyo Seiyaku), Lindane (Mitsui Kagaku, Omuta). Lindane( $\gamma-\mathrm{BHC}$ 99.9\%) added to Polyethylene glycol in a beaker is heated and perfectly dissolved in a water bath; the solution is left standing

TABLE 1

Preparation of 5\% Lindane Ointment

$\begin{array}{lc}\text { Lindane } & 10 \mathrm{~g} . \\ \text { Polyethylene glycol } & 60 \% \\ \quad \text { (Compound 2200) } & \\ \text { Hydrophilic ointment } & 130 "\end{array}$


at room temperature to cool down and solidified; it is next mixed with hydrophylic ointment, agitated with a glass rod, and further agitated and pounded first in Ishikawa's mortar and then, three times or so, on a roller till lindane becomes unformly mixed with the ointment. The Lindane cream thus obtained is pare white in color and mildly aromatic. It gives no color to the skin, nor is it sticky. It is pleasant to the touch. The concentration of $\gamma$-BHC in the cream was kept at $1-2 \%$ when applied to scabies, and at $5 \%$ when applied to trichophytosis as in the case the toxicity must be taken into account in determining the effectual concentration.

II. Effect. As shown in Table 2, the cases chosen for treatment were mainly those of trichophytosis, persisting more than one year in all cases, three years or more in half the cases, 7-10 years in some, over 10 years in a few. The table shows that the drug had a remarkable effect on trichophytosis of the head and face in all cases, a remarkable or noticeable effect on eruptive maculated trichophytosis and eczema marginatum in all cases but one (it had no effect in one case of either disease). The drug had to be rubbed in three or several times a day to have the desired effect on Trichophytia ponpholiciformis. Application once a day proved insufficient. In the hottest season the treatment had to be continued for 3 weeks or more.

TABLE 2

Treatment of skin diseases with $5 \%$ Lindane Ointment

\begin{tabular}{lccc}
\hline \multicolumn{1}{c}{ Patients } & Total & Cured & Failured \\
\hline Trichophytia capillitii & 6 & 6 & 0 \\
Trichophytia facialis & 8 & 8 & 0 \\
Trichophytia maculo-vesiculosa & 12 & 11 & 1 \\
Eczema marginatum & 11 & 10 & 1 \\
Trichophytia ponpholiciformis & 26 & 23 & 3 \\
Trichophytia unguium & 2 & - Treating & \\
Pityriasis versicolor & 5 & 5 & 0 \\
Vitiligo & 5 & 0 & 5 \\
Keratodermia tylodes & 1 & 1 & 0 \\
$\quad$ palmaris progressiva & & & \\
Xeroderma pigmentosum & 1 & 0 & 1 \\
\hline
\end{tabular}

In the treatment of Trichophytia ponpholiciformis a red bean-sized portion of the drug smeared on the affected area with a finger-tip had this effect in 3 days or so. The moisty and eroded skin had its epithelial layers loosened and became dry; the squamae and small eruptions disappeared by degrees and the subjective symptoms such as an itching sensation became reduced and lost.

In 5 cases of Pityriasis versicolor the drug smeared on the affected area and its surrounding area several times at intervals of 3-7 days improved the condition, the subjective 
symptoms and the macules characteristic of the disease disappearing altogether.

The drug was used in the treatment of skin diseases other than trichophytosis, such as Progressive keratodermia of the palm, leucoderma vulgaris and pigmentary xeroderma, and had a curative effect on keratodermia after an itching course of its action. The effect on this diseases is to be ascertained in more detail in a large number of cases. The drug had no effect at all on leucoderma vulgaris or pigmentary xeroderma, probably because abnormality of the vegetative nerve system and the presence of congenital predisposition are responsible for the development of these diseases.

The course of trichophytosis of the finger nails in 2 patients, brother (28 years old) and sister (24), was as follows: The sister had, when she was 16, an eruptive erosion unbearably itching between the first and second fingers of the right hand. It soon spread as far as the 3rd joint and some time later the nail of the first finger became deformed by degrees. All the finger of the left hand as well those of the right became affected and deformed. About this time the brother had all the finger nails of the right hand affected and deformed and got them all removed at our dermatological clinic. The local application of the new drug, commenced on September 5 of the year and continued for a month, produced no change in the condition, microscopic examination made one month after the treatment revealing the presence of causative fungi in the nail fragments dissolved in a $20 \% \mathrm{KOH}$ solution. The treatment (application repeated thrice a day) was resumed accordingly and the effect is under examination.

Some of the above-mentioned cases of trichophytosis are described below.

Case 1. K.-, a 41-year-old female, with chronic Trichophytia ponpholiciformis. Small eruptions, somewhat reddened and giving an itching sensation; were produced 3 years ago between fingers and diagnosed as symptom of Trichophytia ponpholiciformis by a dermatologist. The disease spread into the palms of both hands despite of treatment. The epithelium of the back and interdigital part of the fingers became eroded and loosened. The eruptive parts of the palm gave an increased sensation of itching and the skin became split during the following three years. The BHC treatment was started early in August of the year and continued for some 10 days, rubbing the ointment in 3 times a day. All the symptoms-erosion and reddening of the epithelium, eruption on it, an itching sensation - disappeared, leaving only an eruption on the tip of the first finger of the right hand. After several days, local application continued, the remaining eruption became completely absorbed and lost. No lesion was noted during the subsequent three months.

Case 2. H.-, a 21-year-old female, a case of trichophytosis of the face. A smallfinger-tip-sized white, itching squamous macula was produced on the forehead one year and three months ago. It spread in all directions and an intolerable itch was felt all over the face, especially at night. The diagnosis of trichophytosis was made at the dermatological 
clinic of a certain University hospital. Dietary cure and treatment with Restamin, Vitamin $\mathrm{B}_{6}$, and ultrared-ray radiation were started and Penicillin, Terramycin and Oronein were smeared on the affected area when it was further eczematized. The condition became worse inspite of all these treatments and scratching at night was an additional cause of the aggravation. Hormone and fungicidal therapy had no effect either. The BHC therapy was started at this juncture and continued for 3-4 days: some $1 \mathrm{~g}$. of the drug was thinly smeared on the face before bedtime. The first effected noted was the loss of the itching and drying sensation and the next the disappearance of the superficial gloss and that of the branny squamae. The use of soap, which had to be avoided before BHC therapy, had no unfavorable effect after it.

Case 3. M.-, a 29-year-old female, a case of eczema marginatum. The disease, developed 2-3 years before on the abdomen and armpits, spread in all directions, and the itching sensation it caused kept the patient awake at night from time to time, despite medical treatment and the use of patent medicines. The BHC therapy-local application of the drug 2-3 times a day - was started at the beginning of August of the year and continued for 15-16 days. The result was the reduction and final loss of the itching sensation, the fading of the color of the affected area, and the disappearance of papules, small eruptions and squamae. The treatment continued for another one week eliminated all the symptoms.

Case 4. N. -, a 28-year-old female, a case of progressive Keratodermia of the finger and palm. The patient was readily subject to frostbite in infancy and childhood. Jaundice for which she was treated for 2 months when she was 20 years old, was the only disorder she had suffered from. In November of the year when she was 22, washing and scubbing done after her first delivery was followed by fissure of the ventral surface of the lst joint of the right thumb. All the fingers of the right hand were put in the same condition by degrees, causing pain and hemorrhage and incapacitating the patient for kitchen work. The ointment and vaseline used at home failed to soften the skin. 2 or 3 years later all the fingers and palms of the right hand began to be fissured all over during the winter months and no finger print was to be taken. No remedy used availed nothing. The skin fissure was seen on the left hand too in the winter of 1951. The use of soap always made the condition worse. Thickening and cornification of the skin made the joints of the right hand almost inflexible, and the nails became drawn toward the palmal side and looked like so many beaks. The condition got better and worse by turns in the meantime. The skin more severly fissured, cornified and thickened than befre became flared up when brought in contact with the soap used in washing and gave a burning sensation of pain. Examination at the beginning of August showed: The skin of the fingers and palm of the right hand were fissured all over; the parts made squamous were white like wax and 
loosened; all joints bended toward the palmal side; the skin of the left hand was also fissured and thickened, though not so severely as that of the right. Treatment was started on the day of examination: The BHC preparation was smeared in small doses on the cornified parts of the skin 2 or 3 times a day; Neo-Minophagen $\mathrm{C}$ given by injection and Aderoxin perorally, besides.

Thin scaly skin began to come off in some 4 days; the itching sensation a little alleviated and hard skin came off revealing a wrinked surface behind it in 5-6 days; the fingers began to be easily flexible, nearly all the epithelium came off to be replaced by red-colored skin on the palm in 7 days; the new skin lost some of its red color and a clear finger print was obtainable in 10 days; the only skin fissure left was a faint line on the tip of the first finger and the disease was practically cured in 32 days, the skin softened, the sense of touch normalized, the fingers made sufficiently flexible at the joints and the sweat excreted normally. The remote result of the BHC treatment is under investigation.

III. Summary and Conclusion. A cream containing $99 \%$ or more purified $r$-BHC, Polyethylene glycol and hydrophilic ointment was found to have a fairly good effect on chronic trichophytosis, even on eruptive trichophytosis hitherto considered difficult to cure. It proved effective on trichophytosis of the head and face, eruptive maculated trichophytosis, eczema marginatum and pityriasis versicolor. The therapy appears well worth trying.

The effectiveness of the preparation on these skin diseases is explicable from the fungicidal and fungal-growth-inhibitory action of $\gamma$-BHC experimentally confirmed by the author a few years ago and accepted by the Dermatological Department of Kyushu University Medical School.

For application to a disease like eruptive trichophytosis which has its causative agent deep under the skin surface the drug must be highly permeable. The use of polyethylene glycol which permeate easily through tissue seems to have made the drug so effective on the disease. Local application of this drug, intraadministration of Neo-Minophagen and peroral administration of Aderoxin-these three given in conjunction in one case had a remarkable effect on progressive keratodermia of the fingers and palm. This therapy, however, had no ready effect on trichophytosis of the finger nail even when continued for one month, and had no effect at all on trichophytosis vulgaris or pigmented xeroderma.

The drug has neither irritated nor poisoned. The skin in any case, nor sensitized any patient either.

This work was directed by Prof. Dr. H. Mrzushima, and performed in the Department of Hygienics and Public Health, Kyushu University Medical School, Fukuoka, Japan.

This work has been published previously on the Japanese Journal of Clinical and Experimental Medicine, Vol. 31. No. 3, 1954. 


\section{REFERENCES}

1. Shirakawa, M.: Treatment of parasitic Dermatological Diseases with Hexachlorocyclohexane (BHC) and Experimental Study. Fukuoka Acta Medica. Vol. 42, No. 2, 13-24, 1951. (in Japanese).

2. HiguchI, K.: Recent Advance of the Treatment in Dermatological Field. The Japanese Journal of Clinical and Experimental Medicine. Vol. 29, No. 6, 9-17, 1952. (in Japanese).

3. Morimoto, T.: A Clinical Experience of Some New Fungicidal Ointments. The Dermatology and Urology. Vol. 13, No. 3, 50-55, 1951. (in Japanese).

4. Noma, Y. et al.: On the Solbase, a Watersoluble Ointmentbase. The Dermatology and Urology. Vol. 14, No. 4-5, 55-59, 1952. (in Japanese)

5. ONo, et al.: Application of Polyethylene Glycol in Dermatological Field. Sogo-Igaku. Vol. 8, No. 13, 24-27, 1951. (in Japanese) 